N. E. Piskunov, W. W. Weiss, D. F. Gray, eds.

\title{
Non-local Convection Models for Stellar Atmospheres and Envelopes
}

\author{
F. Kupka ${ }^{1,2}$ \\ Astronomy Unit, School of Mathematical Sciences, Queen Mary, \\ University of London, Mile End Road, London, E1 4NS, U.K.
}

\begin{abstract}
.
We present an overview of the concepts underlying advanced nonlocal Reynolds stress models of turbulent convection and review a comparison of this approach with a series of numerical simulations of fully compressible convection. We then discuss results from applications of the model to complete envelopes of A-type main sequence stars. The nonlocal model reproduces surface velocities in agreement with the lower limit of observed macro- and microturbulence velocities of A-star photospheres, the asymmetry of the surface velocity field as inferred from spectral line profiles, and the overall structure of the photospheric and subphotospheric convection zones, as predicted by the most recent numerical simulations available for these stars. Traditionally, local models of convection are unable to do so. We conclude with a brief survey of extensions of the model which are interesting for other applications such as atmospheres of solar type stars and overshooting below deep convective envelopes or above the core in massive stars.
\end{abstract}

\section{Introduction}

Stellar convection has remained one of the greatest challenges to theoretical astrophysics ever since the era when the first successful model for it had been devised, the now classical mixing length theory (MLT, Biermann 1948). The dramatic progress in quality and amount of observations, the improvements in accuracy and completeness of microscopic data such as opacities, and finally the ever increasing computer power available to astrophysicists have changed the demands on models of convection. For many problems in stellar astrophysics, order of magnitude estimates to reconstruct the approximate thermal structure of a star are now insufficient. A more predictive formalism is required which can be used to compute velocity distributions, thermal profiles, and mixing efficiencies without calibrating numerous parameters each time an individual star or a group of stars is analysed. Two different methodologies have been proposed to meet this challenge: numerical simulations of convection and a new

\footnotetext{
${ }^{1}$ Institut für Astronomie, Universität Wien, Türkenschanzstraße 17, A-1180 Wien, Austria

${ }^{2}$ Institut für Mathematik, Universität Wien, Strudlhofgasse 4, A-1090 Wien, Austria
} 
generation of turbulence models which aim at a "non-local" approach to stellar convection, but at much more modest demands on computational resources.

Each approach has its virtues and its shortcomings. Applications of the numerical simulation approach to stellar convection are limited by the available resources including both computing power and efforts to evaluate the numerical data. For the time being, direct numerical simulations (DNS) which resolve all scales relevant to the flow can only be afforded assuming idealised microphysics. Therefore, simulations of stellar convection have to rely on volume averages over the effects of scales smaller than the grid resolution and their interaction with the larger, resolved scales carrying most of the energy (large eddy simulations, LES). Such simulations can now be performed for stellar atmospheres and the upper part of the envelope (down to the ionisation zone of He II) on a routine basis (cf. the examples quoted in Kupka \& Montgomery 2002). The most sophisticated ones such as those by Stein \& Nordlund (1998) have been performed in 3D and are devoted to individual stars while more extended computations over the HR diagram thus far have been limited to 2D (e.g., Freytag et al. 1996). Complete stellar models cannot be build from such an approach, as the time scales required for thermal relaxation towards a self-consistent solution are too long (see, for example, Kupka 2001). Even if simulations are matched on top of MLT based stellar models, this does not provide us with a solution for problems related to the deeper envelope, such as overshooting (OV) below a deep convection zone, or chemical composition mixing. Concerning stellar atmospheres, a more efficient approach is required wherever large amounts of models and flux calculations are needed, such as population synthesis or complete abundance analyses of groups of hundreds of stars (Heiter \& Luck, these proceedings). A more extreme, but very important example are the $\sim 100$ million stars for which spectra are expected from the IR spectrograph of the GAIA mission. These numbers speak for themselves already, if we merely consider a fully automated analysis using traditional "1D" model stellar atmospheres based on an MLT treatment of convection. Even for an everyday stellar abundance analysis an improved, more realistic convection model would be of convenience for the next couple of years. The need for post-MLT convection models has thus nothing lost of its urgency, despite the success of recent numerical simulations also documented in these proceedings.

\section{Physics of non-local convection models}

Local and non-local models of convection are both based on ensemble averages of the Navier-Stokes equations (NSE). The goal of any convection model is to compute only the moments or correlation functions of solutions to the NSE. But due to the non-linearity of the equations for flows with high Reynolds number and the non-locality of their solutions, no such model has yet been derived without additional statistical assumptions. Derivations of closed systems of equations for the moments are confronted with an infinite hierarchy of equations: each moment $A\left(x_{1}\right)$ is a function of moments of higher order. Hence, an equation for instance for the Reynolds stress $\overline{u_{i} u_{j}}$, where $u_{i}$ and $u_{j}$ are velocity fluctuations around the ensemble mean, depends on quantities such as $\overline{u_{i} u_{j} u_{k}}$. A similar functional dependence on moments of even higher order is observed, for 
example, in equations for $\overline{u_{i} u_{j} u_{k}}$, which causes an infinite loop. This property is a direct consequence of the non-linearity of the NSE. For turbulent flows, where the non-linear (advection) terms dominate over the linear (viscous, etc.) terms, linearisations cannot be used to derive useful convection models, except for particular aspects thereof such as stability analyses (Schwarzschild criterion, etc.). Hence, there is no alternative known around making additional statistical hypotheses, the closure assumptions, if we want to obtain a complete, welldetermined set of equations describing the lower order moments of the NSE for stellar convection.

The matter is complicated by the fact that for an inhomogeneous flow, including turbulent convection, the equations for moments given on one location, say $\overline{u_{i} u_{j}}$, directly depend on values of the moments at another location through derivatives such as $\partial\left(\overline{u_{i} u_{j} u_{k}}\right) / \partial r$. To account for that property turbulent convection models have to be non-local, as opposed to the classical local convection models which provide the convective flux $F_{\text {conv }}$ as a function of local mean values and just the mean temperature (and pressure) gradients alone. The most well-known example among the local convection models is MLT. Non-local convection models on the other hand provide differential equations for low order moments such as $F_{\text {conv }}$ and are closed with assumptions on moments of higher order. Consequently, quantities such as $F_{\text {conv }}$ or $\overline{u_{i} u_{j}}$ explicitly depend on the function values of other moments at different (neighbouring) locations. Some problems in turbulent convection such as the overshooting of fluid into stably stratified layers in stellar atmospheres can only be addressed by a non-local approach. Others such as mixing or transport of angular momentum benefit from a non-local approach to turbulent convection as well, particularly, because the boundary regions of a convection zone are important in their case. Local models perform notoriously poor within these regions (cf. the discussion in Canuto 1993).

Two main schemes for deriving closed equations of non-local models have been used thus far. One-point closure models consider averages of the NSE in physical space. The more advanced among them also compute various components of or sums over $\overline{u_{i} u_{j}}$ and are known as Reynolds stress models. Such models have first been proposed for turbulent stellar convection by Xiong (1978, 1985). More updated versions, which still include a mixing length to compute the dissipation rate of turbulent kinetic energy, were given by Xiong et al. (1997) and by Grossman et al. (1993). Canuto (1992, 1993, 1997) suggested the first models which avoid the use of a local scale length (mixing length). Hence, they may be called fully non-local models for stellar convection, where non-locality refers to the equations for the second order moments (convective flux, turbulent pressure, turbulent kinetic energy and its dissipation rate, and mean temperature fluctuations), as the closure relations inevitably introduce locality assumptions on the higher order moments. Two-point closures on the other hand are based on ensemble averages performed in Fourier space and provide the spectra of the moments. Integration over these spectra yields the one-point averaged quantities required in stellar modelling. Canuto \& Dubovikov (1998, CD98) have used this approach to rederive an improved version of the earlier Canuto (1992) model, again avoiding the use of a mixing length. 
Their approach is based on a general turbulence model they have developed which thus far has successfully passed about 100 tests made for various types of flow. The tests included mostly laboratory and numerical simulation data (see CD98). The "CD98 model" is a non-local Reynolds stress model of turbulent convection. It consists of five differential equations for the horizontal averages of the second order moments which have to be solved together with the stellar structure equations. To the latter they are coupled through the fluxes of enthalphy (convective flux) and turbulent kinetic energy, and through the turbulent pressure. Due to its formal similarity with the previous models of Canuto (1992, 1993) the CD98 model can easily be extended to include compressibility effects beyond the Boussinesq approximation, anisotropy, and non-locality. In the form given by (Canuto 1992, 1993) the model has been successfully tested for geophysical flows (Canuto et al. 1994, 2001). Despite these reassuring results there is of course no guarantee that this approach works equally well for the case of stellar convection. Although the model of Xiong et al. (1997) has a number of structural similarities with the CD98 model (or actually, from the viewpoint of its derivation, the model of Canuto 1992) and despite it has already been applied to the stellar case (see also these proceedings), the differences to the models of Canuto $(1992,1993$ ) and CD98 (which, e.g., avoid the use of a mixing length) are too large to wave independent and thorough testing. In addition, a comparison to numerical simulations of compressible convection has not been done before for any of the advanced Reynolds stress models proposed for stellar convection. To avoid that shortcomings of the modelling of the hydrodynamics are confused with other problems (opacity data, non-locality of radiative transfer in stellar atmospheres, etc.), a step-by-step procedure for the application and tests of this type of model was suggested in Kupka (1996). The idea was to first test the models with numerical simulations of compressible convection with idealised microphysics and later on relieve these limitations for the computation of realistic stellar models. The final goal is to leave the parameter space nowadays accessible to the simulations and apply a thoroughly tested and physically much more complete model to problems where, despite their much more severe limitations, MLT and phenomenological models have been the only choice thus far.

\section{Testing non-local models with numerical simulations}

In Kupka (1999a, 1999b, 2001) the CD98 model was compared to 3D numerical simulations of fully compressible convection performed by Muthsam et al. (1995, 1999) and to more recent computations by Muthsam. The latter encompass their predecessors by a higher numerical resolution, usually lower Prandtl numbers, and, in some casees, higher convective efficiencies (Kupka \& Muthsam 2003 and other publications of Muthsam). The simulations have been performed for plane parallel geometry and constant surface gravity, for a perfect gas equation of state with a prescribed ratio $\gamma$ of specific heats (usually 5/3), and a radiative conductivity which varied as a function of depth but was kept unaltered as a function of time. The latter permits to easily define the parameter space (efficiency, stability) for which the simulations take place. Moreover, this simplified microphysics corresponds to how equation of state and radiative transfer are dealt with in the derivation of the convection model. The diffusion approximation is assumed 


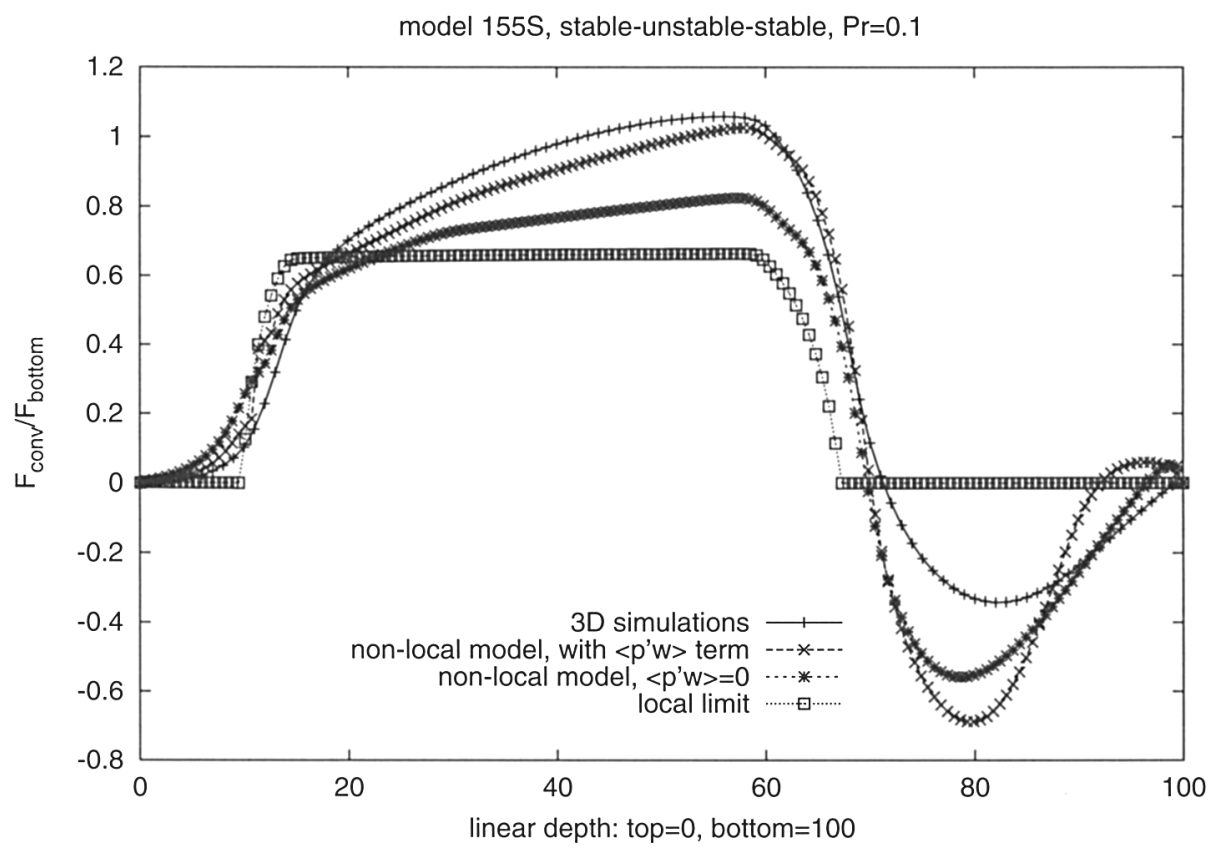

Figure 1. Convective flux in units of input flux at the bottom for a case with efficient convection and a Prandtl number of 0.1 .

to compute the radiative flux $F_{\text {rad }}$. About two dozen configurations have been investigated so far including a convection zone placed between two stable layers, or two convection zones surrounding a central, stable layer. The convective efficiency range includes cases where $F_{\text {rad }}$ is at least $75 \%$ throughout the simulation domain, while for a few other configurations, $F_{\text {conv }} \sim 100 \%$ in some layers within the convection zone. Viscosity was defined by a constant Prandtl number in the range of 0.1 to 1.0 and Rayleigh numbers for these simulations in the centre of the convection zone were between $10^{5}$ and $10^{6}$, thus placing the simulations in the highly turbulent regime (3 to 4 orders of magnitude above the critical Rayleigh number for pure convection with a penetrable boundary). The numerical resolution is $72 \times 50^{2}$ for the earlier simulations, while the more recent ones have been done at $125 \times 100^{2}$ and $160 \times 140^{2}$. The temperature contrast between top and bottom varies between 1:4 and 1:10 while the density contrast ranges from 1:10 to 1:20, encompassing between 4 and 5 pressure scale heights $\left(H_{\mathrm{p}}\right)$. Individual unstable (convection) zones span between 1 and $3 H_{\mathrm{p}}$ in these simulations. Thus, the parameter range shares many similarities to that one encountered in the envelope of A stars in layers from the centre of the $\mathrm{H}$ convection zone to below the region where He II becomes fully ionised.

The evaluation procedure for the calculations has been briefly explained in Kupka (1999b, 2001). Both simulations and the equations of the non-local 
model 3J, stable-unstable-stable, $\mathrm{Pr}=1$

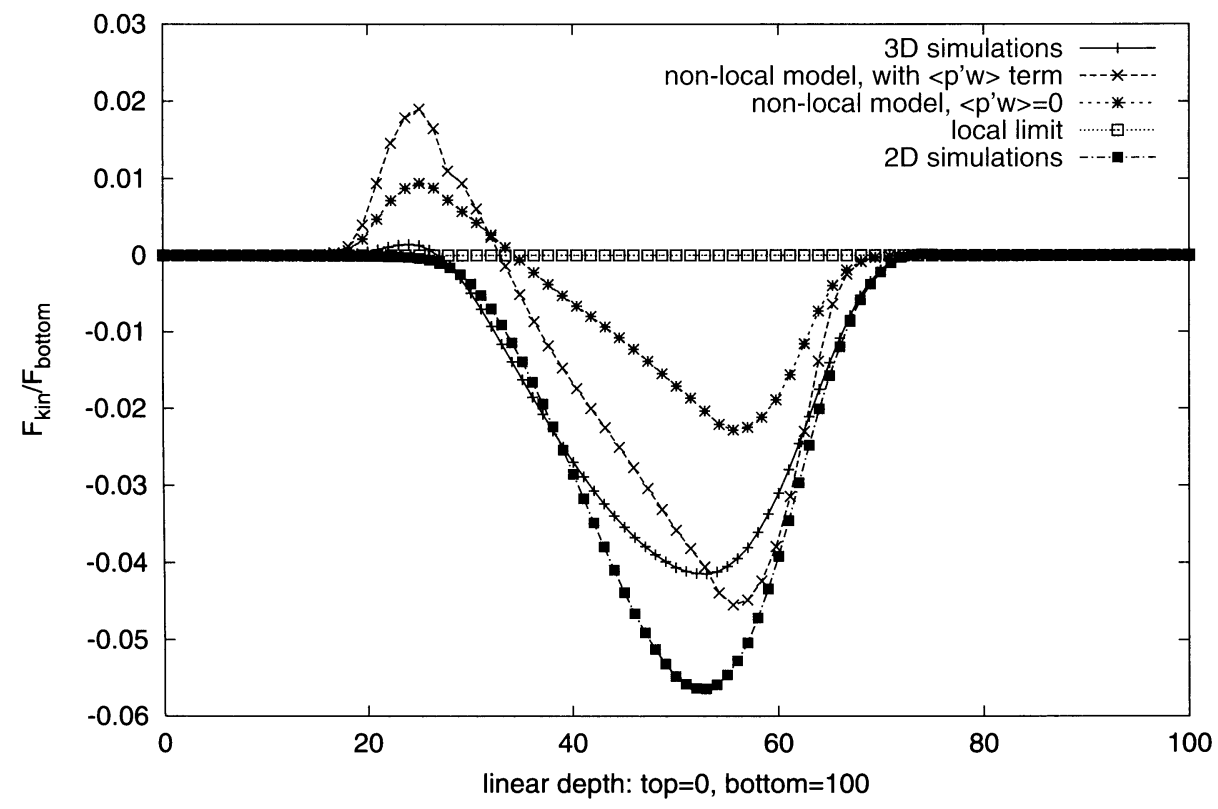

Figure 2. Flux of turbulent kinetic energy in units of input flux at the bottom for a case with ineffecient convection and unit Prandtl number.

convection models are integrated in time over the thermal (Kelvin-Helmholtz) time scale $t_{\mathrm{KH}}$, the longest one in the problem. The moment equations attain a stationary state after $t_{\mathrm{KH}}$ at which the simulations can be averaged over time to calculate moments without including transient states. Reliable results for higher order moments, in particular those including pressure fluctuations, have to be computed from samples distributed over a significant fraction of $t_{\mathrm{KH}}$, while the mean structure can be derived within merely one convective turnover time $t_{\mathrm{c}}$, equaling a few multiples of the sound crossing time $t_{\mathrm{s}}$, the shortest time scale in the problem. We give three examples here. Figure 1 shows $F_{\text {conv }}$ in units of the input flux at the bottom for model " $155 \mathrm{~S}$ ". In this case, a vigorously convective region of $2.5 H_{\mathrm{p}}$ is embedded between two stable layers. The results corroborate an earlier example discussed in Kupka $(1999 \mathrm{~b}, 2001)$ in which $F_{\text {conv }}$ never exceeds $\sim 25 \%$ : the local model fails near the boundary to stable stratification and in the OV region while the non-local model reproduces the behaviour found by the 3D simulations not only qualitatively, but even quantitatively to at least within a factor of 2 , and frequently even within $20 \%$. To a large extent the differences to the simulations observable in Fig. 1 can be traced to the modelling of the pressure flux, i.e. the correlation $\overline{p^{\prime} w}=\left\langle p^{\prime} w\right\rangle\left(p^{\prime}\right.$ and $w$ are fluctuations of pressure and vertical velocity around the ensemble mean). Evidently, even the standard closure proposed in Canuto (1993), $\overline{p^{\prime} w}=-0.2 \bar{\rho} \overline{q^{2} w} \approx-0.4 F_{\text {kin }}$ with $F_{\text {kin }}$ as the flux of $K=\overline{q^{2}} / 2$, the turbulent kinetic energy, is a major improvement for the model compared to the case in which this term is neglected 
$\left(\overline{p^{\prime} w}=0\right)$. Fig. 1 also shows that the performance of this closure in the OV region is not as satisfactory as it is in the interior of the convection zone. Unfortunately, this term cannot be derived from the Canuto-Dubovikov turbulence model. One interesting aspect about comparisons done with "model $155 \mathrm{~S}$ " is that the moments computed from its simulation are less constrained by the definition of the radiative conductivity than cases with lower convective efficiency (as in Kupka 1999a,b). Hence, the modelling of non-local quantities such as $F_{\text {kin }}$ becomes more important. The latter attains $-40 \%$ for the case shown in Fig. 1 as compared to $-4 \%$ in the model of inefficient convection, " $3 \mathrm{~J}$ ", studied in Kupka (1999b, 2001). Nevertheless, comparisons for cases with inefficient convection are conclusive just as well. Figure 2 compares $F_{\text {kin }}$ of "model $3 \mathrm{~J}$ " for $2 \mathrm{D}$ and $3 \mathrm{D}$ simulations with the two variants of the non-local model also discussed in Fig. 1 and with the local limit of the CD98 approach. Indeed, also for this scenario the inclusion of $\overline{p^{\prime} w}$ provides an improvement: $F_{\text {kin }}$ is much closer to the 3D simulation result, if this term is taken into account. Moreover, $F_{\text {conv }}$ from the improved non-local model falls short of the 3D simulations by only $1 \%$ inside the convectively unstable region and outperforms the $2 \mathrm{D}$ simulations for this scenario. This has to be compared with the several percent found in previous work (Kupka 1999b, 2001). Local models of course cannot provide this quantity. Hence, the local limit of the CD98 model also predicts $F_{\text {kin }}$ to be zero. In Kupka (2001) it was noted that the 2D simulations overestimate the OV while the non-local models rather underestimate it for the cases studied. $F_{\text {conv }}$ was found continuing much deeper into the lower stable zone instead of falling off as observed in the 3D case and the non-local model (which predicted an even faster decay). Figure 2 shows that the 2D simulation overestimates $F_{\text {kin }}$ in the centre of the convection zone but otherwise performs very well, provided we do not consider OV regions where this quantity falls below $0.1 \%$. This complements the comparison between the 2D and the 3D case of this model given in Muthsam et al. (1995) where the kinetic energy of the flow was found to be twice as large in $2 \mathrm{D}$ than in $3 \mathrm{D}$.

Figure 3 emphasises that the differential equation for the dissipation rate of turbulent kinetic energy $\epsilon$ is an important ingredient for the non-local convection models. It avoids the introduction of a scale length (mixing length) $l$ defined by the assumption $l \approx 0.8 K^{1.5} / \epsilon$, where $K$ is given by another relation (a differential equation in case of non-local models). Here, we have considered the case in which $\overline{p^{\prime} w}=0$, which has also been assumed in Kupka (1999b, denoted ApJL 526 in Figure 3). The scale lengths $l$ derived from the non-local models are in overall agreement with the value obtained from the simulations. The difference between the two non-local models is due to the more refined closure used for the third order moments taken from Canuto et al. (2001) which had not been available to Kupka (1999b). It is of the same magnitude as the difference to the simulations. If we use the closure for $\overline{p^{\prime} w}$ described above, $l$ is overestimated inside the convection zone by $40 \%$ to $50 \%$, though this is still smaller than the overestimate of $80 \%$ resulting from 2D simulations of this scenario. Nevertheless, all these calculations predict a rapid decrease of $l$ within the OV. The assumption of $l$ to be proportional to $H_{\mathrm{p}}$, however, as done in most astrophysical applications and particularly in MLT, begins to fail near the boundary to stable stratification and is completely off the mark in the OV region, especially for the layers below 
model 3J, stable-unstable-stable, $\mathrm{Pr}=1$

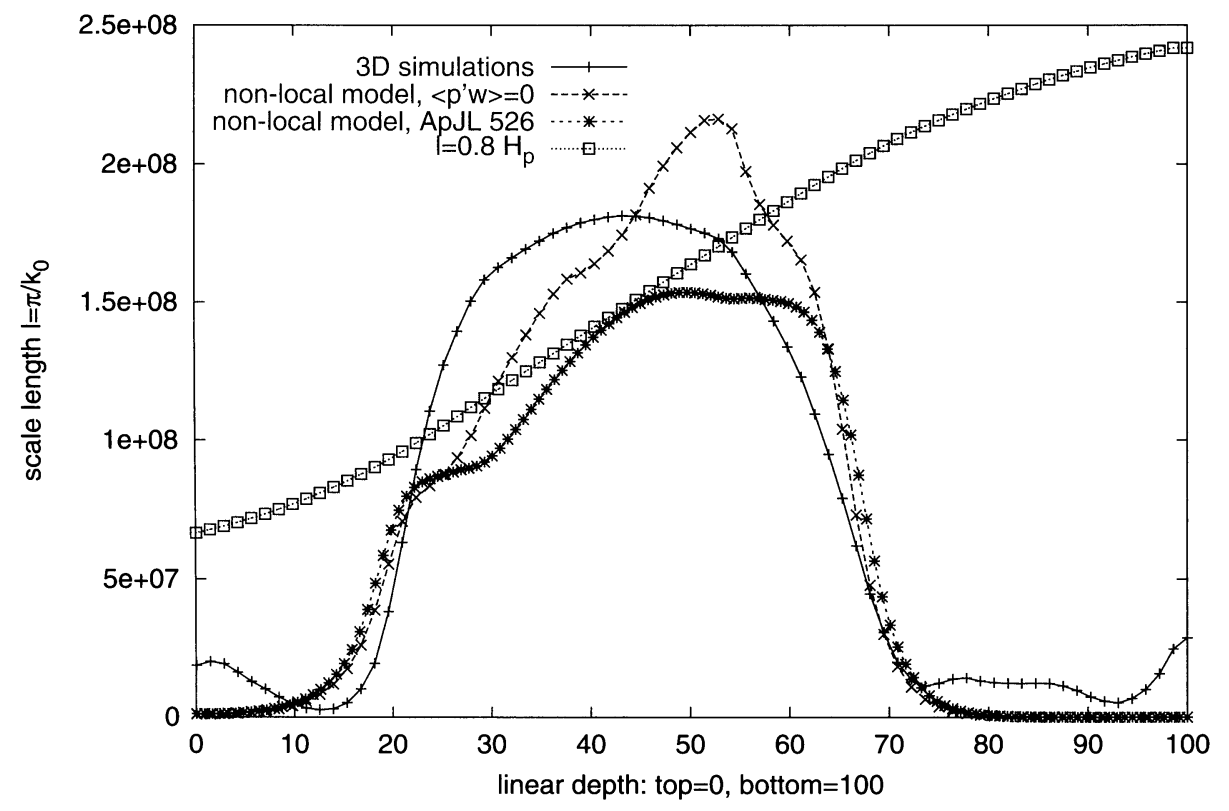

Figure 3. The scale length $l=\pi / k_{0} \approx 0.8 K^{1.5} / \epsilon$ computed from $3 \mathrm{D}$ simulations and non-local models (different closures) for a case with inefficient convection. The standard mixing length rule fails near the boundary of the convection zone and in the OV region.

the convection zone (we have set $\alpha=0.8$ to fit the computed $l$ in the centre of the convection zone).

The most important conclusions drawn from comparisons with idealised simulations of compressible convection, which will be discussed in detail in Kupka (2003) and Kupka \& Muthsam (2003), can be summarised as follows: a) the usage of the most complete version of the Canuto $(1992,1993)$ and CD98 models is essential for the modelling of the hydrodynamics, b) the improvements of local models are most obvious near the boundary to stable stratification and within the OV region, c) it is essential to use a differential equation for $\epsilon$ to avoid usage of the ill-defined scale length $l$ and its related tuning parameter(s), d) the tuning of various closure parameters is a fruitless exercise: although a fine tuning may allow a precise match of a particular quantity (such as the solar radius), tweaking numerical parameters is normally a waste of time, because the failure of a closure relation is generally due to its functional form rather than a numerical factor. Hence, an improvement of the entire model could only be achieved by considering a physically more realistic closure hypothesis. 


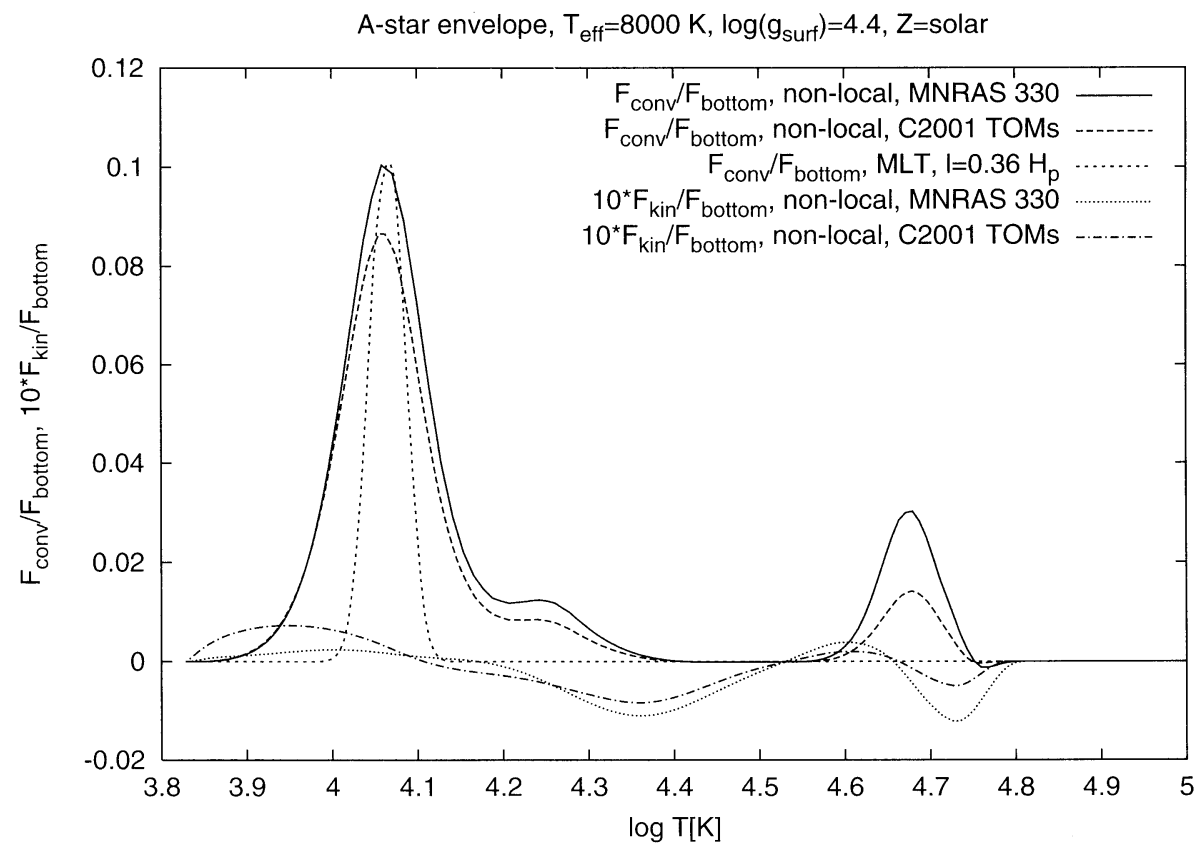

Figure 4. $\quad F_{\text {conv }}$ and $10 F_{\text {kin }}$ in units of the bottom input flux for an envelope with $T_{\text {eff }}=8000 \mathrm{~K}$, surface $\log (g)=4.4$, and solar metallicity. The relations for the third order moment solved in Kupka \& Montgomery (2002), MNRAS 330, are based on a modified version of the original proposal of Canuto et al. (2001), C2001 TOMs. Such alterations only result in minute changes compared to the totally different solution obtained from MLT (tuned to match $\max \left(F_{\text {conv }}\right)$ ).

\section{Envelopes of A stars: comparison with data and with local models}

After the promising comparisons with numerical simulations based on idealised microphysics the computer code to solve the equations of non-local convection models was modified to calculate stellar envelopes with realistic microphysics. First results for the ionisation zone of $\mathrm{He}$ II in A-stars were presented by Kupka \& Montgomery (2001). Fully self-consistent models for the complete, convective part of A-star envelopes ranging from the upper atmosphere down to below the lower overshooting region of the He II convection zone were presented by Kupka \& Montgomery (2002). The necessary alterations to the original code primarily consisted in the inclusion of the Los Alamos equation of state and OPAL opacities (Rogers et al. 1996, Iglesias \& Rogers 1996) and the adoption of a spherical geometry and an adaptive grid based on the surface mass as an independent variable. The boundary conditions have been altered to keep temperature, pressure, and radius fixed at the top, while the luminosity is kept fixed at the bottom. With this setting, envelopes computed with the code described in Pamyatnykh (1999) can be used as initial solutions. Self-consistency 
(independence of the convection model used to compute the initial solution) is guaranteed by placing the domain for which the envelope is calculated into sufficiently thick stable boundary layers, relaxation over $t_{\mathrm{KH}}$, and cross-checking the final solution for being radiative near the boundaries. Photospheric radiative transfer is treated in the grey (diffusion) approximation apart from an optional "dilution factor" for compatibility with the upper boundary condition provided by the code described in Pamyatnykh (1999) and the effect of smoothing of temperature fluctuations by radiative transfer (Spiegel 1957). Further details are given in Kupka \& Montgomery (2002). The model stellar envelopes consist of usually 200 mass shells distributed from $\tau_{\text {Ross }} \sim 10^{-3}$ in the photosphere down to regions where $T \sim 10^{5} \mathrm{~K}$. The relative sizes of the shells are re-adjusted during the solution procedure to resolve the temperature gradient.

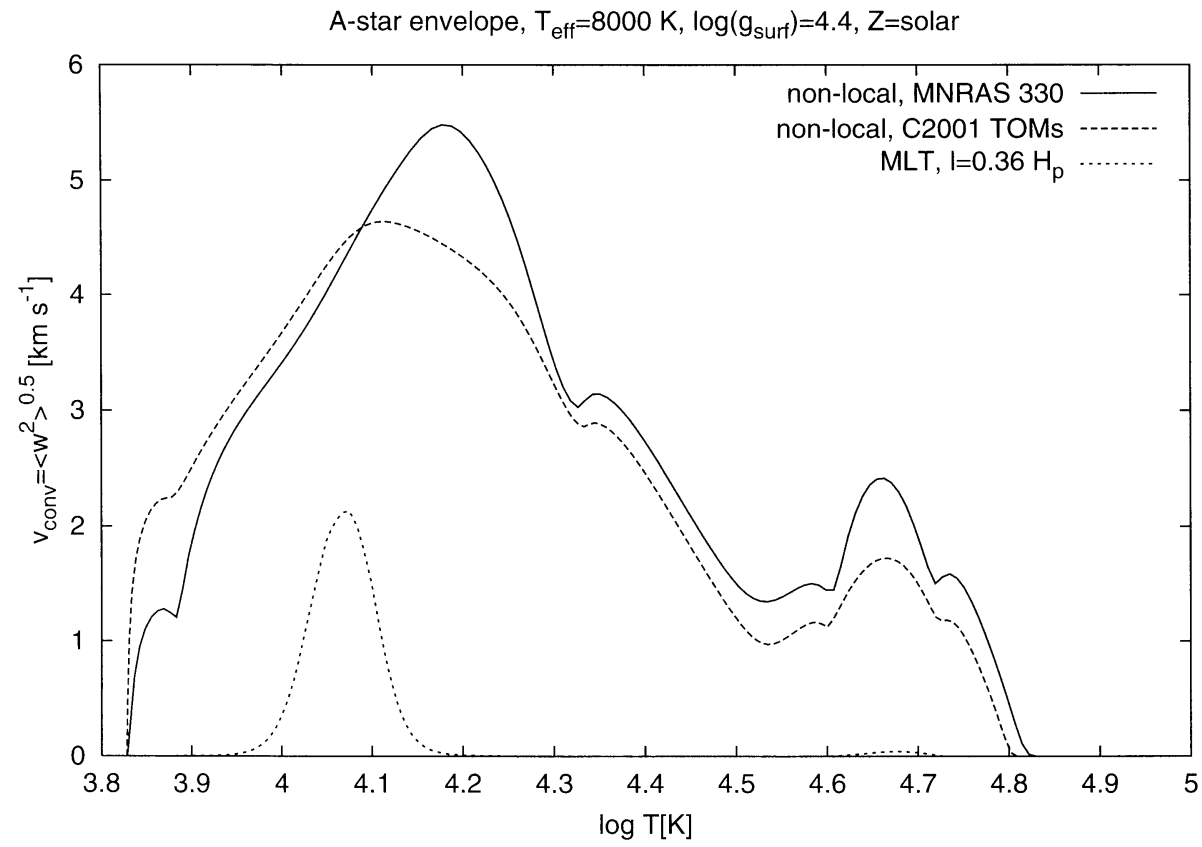

Figure 5. Average convective (root mean square) velocities for the same envelope as in Figure 4. Again the difference between the same particular closures of the non-local model is minute compared to the one obtained from MLT (tuned to match $\max \left(F_{\text {conv }}\right)$ ).

Calculations were done for a number of variants of the non-local convection models, but for their presentation Kupka \& Montgomery (2002) selected the model which had been found to compare best with the numerical simulations for idealised microphysics thus far. Envelopes were computed for a range of metallicities $(\mathrm{Z}=0.006,0.02$, and 0.06$)$, for iso-sequences of constant surface gravity $\log (g)$, and for models along an evolutionary track of a star with $2.1 M_{\odot}$. Here, we summarise their main results along with a few additional remarks. First of all, for stars with $T_{\text {eff }}$ between $7200 \mathrm{~K}$ and $8500 \mathrm{~K}$ in the main sequence band, 
the convection zones due to the ionisation of $\mathrm{H}$ and of $\mathrm{He} \mathrm{I}$ are always merged into one region. This " $\mathrm{H}$ convection zone" and a second zone deeper inside the envelope due to the ionisation of $\mathrm{He}$ II, the "He II convection zone", were found to be connected in terms of the velocity field, but separated in terms of $F_{\text {conv }}$ and temperature fluctuations. In this sense, both convection zones are connected by a single overshooting region in which average (root mean square) vertical velocities never drop notably below $1 \mathrm{~km} \mathrm{~s}^{-1}$. This result is supported by the 2D numerical simulation of Freytag (1995) and Freytag et al. (1996). Local convection models fail on this problem, as they cannot account for overshooting. Moreover, the photospheric velocities found for the non-local model are of the order of 1.5 to $2 \mathrm{~km} \mathrm{~s}^{-1}$ at $\tau_{\text {Ross }} \sim 2 / 3$ which is consistent with the lower limit of observed micro- and macroturbulence velocities (cf. Landstreet 1998) and also the 2D simulations. Again, local models are not able to retrieve this result. Moreover, $F_{\text {kin }}$ is positive in the photosphere and the same naturally holds for the skewness $S_{w}$ of the vertical velocity fluctuations. Following a simple upstream-downstream model of the flow topology as in CD98 this implies that the filling factor, the fraction of the horizontal area covered by upstreaming material, must be less than $1 / 2$, in agreement with the observed line profiles of Astars (cf. Landstreet 1998). Once more, local convection models cannot predict this quantity. Figures 4 and 5 summarise these findings for an envelope model with a $T_{\text {eff }}$ of $8000 \mathrm{~K}$. The overshooting found below the He II convection zone ranges about $0.45 H_{\mathrm{p}}$, if we consider a non-zero convective flux as the criterion for the extent of overshooting (as in Kupka \& Montgomery 2002). A slightly larger result of about $0.6 H_{\mathrm{p}}$ is obtained, if we consider the turbulent kinetic energy $K$ instead of $F_{\text {conv }}$. Beyond that region the moments decay exponentially at a rate determined by the Prandtl number of the problem. This extent of OV is supported by the 2D simulations of Freytag (1995) and Freytag et al. (1996) in the sense of a lower limit, as their calculations predict the well-mixed OV region to be even three times larger in terms of radial depth. Finally, it was noted by Kupka \& Montgomery (2002) that $\alpha$ in MLT models would have to be increased from 0.4 at a $T_{\text {eff }}$ of $8000 \mathrm{~K}$ to about 1.0 at a $T_{\text {eff }}$ of $7100 \mathrm{~K}$ to match the maximum of $F_{\text {conv }}$ in the $\mathrm{H}$ convection zone, while a completely different set of $\alpha$ values, larger than 1.5, would be required to achieve the same in the lower lying He II convection zone inside the envelope. This is all the more interesting, as the $2 \mathrm{D}$ simulations predict exactly the same trends. As can be concluded from the $\alpha$ values for MLT already, the temperature gradients found for A-type main sequence stellar envelopes change from inefficient convection with radiative gradients for a $T_{\text {eff }}$ around $8000 \mathrm{~K}$ to one with adiabatic stratification appearing for values of $T_{\text {eff }}$ just below $7200 \mathrm{~K}$. These results hold also for higher and lower metallicity models, although the exact numerical values change. The robustness of these results towards minor changes in the closure assumptions is illustrated in Figures 4 and 5: if the original model of third order moments of Canuto et al. (2001) is used rather than its modification mentioned in Kupka \& Montgomery (2002), throughout most of the envelope the results change by less than $10 \%$ and exceed $20 \%$ only in the photosphere and the centre of the He II convection zone. Such changes are of the same magnitude as found for the case of idealised microphysics (Figures 1 to 3 ). 


\section{Some further extensions, conclusions}

A direct comparison to the numerical simulations performed for stellar atmospheres and the upper part of stellar envelopes is possible and an obvious next step. However, it is important to do such a comparison for identical microphysics (equation of state, opacitites) and numerical resolution to draw more solid conclusions. The code used in Kupka \& Montgomery (2002) treats only the viscous and conductive (radiative transfer) parts of the problem implicitly, as done in most numerical simulations. Once the fully implicit solver currently being developed and tested is ready for production runs, deep convective envelopes as in $\mathrm{F}$ stars or the sun can be calculated, ranging from the entire photosphere down to below the overshooting region of the lowermost envelope convection zone in these stars. With only a few minor changes the same technique is readily applicable to the problem of overshooting in convective cores of massive stars. At this stage, a more extended comparison with data will be extremely interesting, just as a comparison to the convection model used by Xiong et al. (1997, see also these proceedings).

A number of extensions of the non-local convection models have recently been proposed to include the effects of concentration gradients and a mean flow due to rigid or differential rotations. Although we cannot extend on them here, these will be extremely useful, if the non-local modelling approach is used more frequently in its most promising fields of application: first and foremost stellar evolution (globular clusters, pre-main sequence, late phases of stellar evolution, cluster photometry). Moreover, various aspects of the notorious $\mathrm{Li}$ problem could be addressed with these models, while complete solar models based on this approach could be challenged by results from helioseismology. As for stellar atmospheres, the most interesting applications certainly include the "mass production" areas such as stellar population synthesis. Because this will require the computation of stellar atmospheres with granulation effects at least for the cool parts of the HR diagram, an extension of the model towards a non-local treatment of radiative transfer is most likely necessary. Finally, the application of this approach to cases involving a magnetic field appears very attractive, although it requires that a number of basic theoretical problems have successfully been addressed before.

Acknowledgments. This research was performed within project $P 13936$ TEC of the Austrian Fonds zur Förderung der wissenschaftlichen Forschung $(\mathrm{FwF})$. Support by the UK Particle Physics and Astronomy Research Council under grant PPA/G/O/1998/00576 while writing this contribution is gratefully acknowledged. I thank H.J. Muthsam for permission to use his simulation data.

\section{Discussion}

STEIN: 2D and 3D simulations have very different relation between velocity and temperature fluctuations. As a result $2 \mathrm{D}$ simulations lead to incorrect line profiles (see Asplund, Ludwig, Nordlund \& Stein, A\&A 346, L17, 2000.)

KUPKA: Yes, I agree. This is why I have used the 3D simulations of H. Muthsam rather than their $2 \mathrm{D}$ counter-parts: the latter predict clearly different higher order correlations and too large an overshoot. As far as A stars are concerned, the 
only simulations available to compare to were done in 2D and I would certainly prefer, if 3D simulations were available for at least a few cases. The actual test for the capability of the non-local model to predict line profiles is indeed the comparison to observations such as those by Landstreet 1998, A\&A 338, 1041 once synthetic spectra will be available based on this treatment.

\section{References}

Biermann, L. 1948, Z.Astrophys., 25, 135

Canuto, V. M. 1992, ApJ, 392, 218

Canuto, V. M. 1993, ApJ, 416, 331

Canuto, V. M. 1997, ApJ, 482, 827

Canuto, V. M., Minotti, F., Ronchi, C., Ypma, R. M., \& Zeman, O. 1994, J.Atm.Sci., 51 (No. 12), 1605

Canuto, V. M., \& Dubovikov, M. S. 1998, ApJ, 493, 834 (CD98)

Canuto, V. M., Cheng, Y., \& Howard, A. 2001, J.Atm.Sci., 58, 1169

Freytag, B. 1995, PhD thesis, University of Kiel

Freytag, B., Ludwig H.-G., Steffen M. 1996, A\&A, 313, 497

Grossman, S. A., Narayan, R., \& Arnett, D. 1993, ApJ, 407, 284

Iglesias, C. A., \& Rogers, F. J. 1996, ApJ, 464, 943

Kupka, F. 1996, in Model Atmospheres and Spectrum Synthesis, S. J. Adelman, F. Kupka \& W. W. Weiss, ASP Conf. Ser. Vol. 108, 73

Kupka, F., 1999a, in Theory and Tests of Convection in Stellar Structure, A. Gimenez, E. F. Guinan \& B. Montesinos, ASP Conf. Ser. 173, 157

Kupka, F. 1999b, ApJ, 526, L45

Kupka, F. 2001, in Proceedings of the COROT/SWG Sept. 2000 meeting, E. Michel, Paris, http://dasgal.obspm.fr/ michel/MilestoneProc/SWGproc2000.html

Kupka, F. 2003, ApJ, to be submitted (papers I+III)

Kupka, F., \& Montgomery, M. H. 2001, in Proceedings of the COROT/SWG Sept. 2000 meeting, E. Michel, Paris,

http://dasgal.obspm.fr/ michel/MilestoneProc/SWGproc2000.html

Kupka, F., \& Montgomery, M. H. 2002, MNRAS, 330, L6

Kupka, F., \& Muthsam, H. J. 2003, ApJ, to be submitted (paper II)

Kurucz, R. L. 1979, ApJS, 40, 1

Landstreet, J. D. 1998, A\&A, 338, 1041

Muthsam, H. J., Göb, W., Kupka, F., Liebich, W., \& Zöchling, J. 1995, A\&A, 293,127

Muthsam, H. J., Göb, W., Kupka, F., \& Liebich, W. 1999, New Astr., 4, 405

Pamyatnykh, A. A. 1999, Acta Astronomica, 49, 119

Rogers, F. J., Swenson, F. J., \& Iglesias, C. A. 1996, ApJ, 456, 902

Spiegel, E. A.,1957, ApJ, 126, 202

Stein, R. F., \& Nordlund, Å 1998, ApJ, 499, 914 
Xiong, D. R. 1978, Chin.Astron., 2, 118

Xiong, D. R. 1985, A\&A, 150, 133

Xiong, D. R., Cheng, Q. L., \& Deng, L. 1997, ApJS, 108, 529 